\title{
Strategien zur Erzeugung positiver Heilungseffekte
}

\author{
Thomas Weiß
}

\begin{abstract}
Mit der richtigen Strategie können Therapeuten gezielt Placeboeffekte in ihrer Behandlung einsetzen. Wer seinem Patienten aktiv zuhört, dessen Therapie-Erwartungen berücksichtigt und ihm Mitspracherechte bei der Behandlung einräumt, kann positive Heilungseffekte generieren. Auch ein gepflegte Erscheinungsbild, Empathie sowie ein angenehmes PraxisAmbiente steigern die Zufriedenheit eines Patienten und erhöhen seine Compliance.
\end{abstract}

\section{Placebo-Definition des NIH}

Zahlreiche wissenschaftliche Forschungsarbeiten belegen, dass Placebos ein erhebliches Heilungspotential besitzen. Die aus Placebo-Therapien resultierenden positiven Effekte werden zumeist als „unspezifisch“, „psychologisch“ oder „psychophysiologisch“ bezeichnet [1-3].

Man kann Placeboeffekte allerdings auch anders verstehen. Nach intensiver Diskussion definierte eine Expertengruppe des US-amerikanischen ,National Institute of Health، (NIH) im Jahr 2002 den Begriff Placeboeffekte durch „positive Heilungseffekte“ [4]. Diese ergeben sich aus der Nutzung irgendeiner therapeutischen Maßnahme und/oder kommen durch die symbolische Bedeutung einer Intervention für den Patienten zustande. In gleicher Weise definiert das ,National Center for Complementary and Integrative Health' den Placeboeffekt [5].

Neurobiologie Bei einem Placeboeffekt existiert neben Konditionierungseffekten eine sehr enge Beziehung zwischen dem Induzieren von Erwartungen, der Wirkung auf unterschiedliche Hirnregionen und folglich auf Transmittersysteme [6]. Die Erwartungsinduktion beim Placeboeffekt gehört zu den wenigen psychologischen Phänomenen, welche so gut erforscht und neurobiologisch erklärt werden können.

\section{Klinische Bedeutung}

Die Placebo-Definition des NIH ist aus mehreren Gründen klinisch hoch bedeutsam. Erstens werden Placeboeffekte als eigenständige therapeutische Kategorie definiert, wodurch eine Unterteilung in „spezifische“ und „unspezifische“ Effekte entfällt. Zum Zweiten werden Placeboeffekte als positiv anerkannt. Drittens werden die Effekte theorieunabhängig gesehen. Damit unterstellt die NIHDefinition keinerlei Annahmen zu den Ursachen der Therapiewirkung. Viertes resultieren Placeboeffekte aus

\section{ZUSATZINFO \\ Volkswirtschaftlicher Aspekt}

Placeboeffekte sind volkswirtschaftlich von großer Bedeutung. Einerseits sparen sie kaum abschätzbare Beträge bei der Therapie von Schmerzpatienten ein, andererseits verschlingen Placeboeffekte immense Summen, weil jedes neue Pharmakon seine Effizienz gegenüber Placebos im Doppelblindverfahren belegen muss.

Sicht des Patienten hinsichtlich der Behandlung, woraus sich ihre nachgewiesene individuelle Variabilität ergibt. Und schließlich werden unter dem Begriff ,Placeboeffekt“ neben pharmakologischen Effekten nun auch andere Effekte erfasst - so auch die Wirkung eines Behandlers auf seinen Patienten.

\footnotetext{
Merke

Konsequenzen aus der NIH-Definition

Placeboeffekte repräsentieren einen Bestandteil jeder medizinischen Intervention, weil jede medizinische Intervention eine Interaktion zwischen Therapeut und Patient beinhaltet. In diesem Sinne muss dann unterschieden werden zwischen dem positiven Placeboeffekt und seinem „bösen Zwilling“, dem negativen Noceboeffekt.
}

\section{Einfluss des Therapeuten}

Gemäß der NIH-Definition muss es eine kaum kontrollierbare Menge unterschiedlicher Einflüsse geben, die durch das Wecken spezifischer Erwartungen, Beobachtungen oder Konditionierungsreize - Placeboeffekte hervorrufen können [1]. Eine herausragende Rolle hierbei spielt die Interaktion zwischen Therapeut und Patient. 
Daraus ergibt sich zwangsläufig die Frage, ob nicht die Persönlichkeiten oder Fähigkeiten von Patienten und Therapeuten letztendlich ausschlaggebend für die Ausprägung positiver Heilungseffekte sind.

\section{Erklärungsversuche}

Irrungen Angesichts der breitgefächerten interindividuellen Varianz von Placeboeffekten wurde in der wissenschaftlichen Literatur früher die Hypothese aufgestellt, dass das Ausmaß einer Placebo-Hypalgesie, d. h. einer Reduktion der Schmerzwahrnehmung durch die Gabe eines Placebo-Präparats, von der Persönlichkeit des Patienten abhängt. Viele a priori als wesentlich empfundenen Persönlichkeitsvariablen haben tatsächlich jedoch keinerlei Bedeutung für einen Placeboeffekt. Dazu zählen u.a. demografische Parameter wie das Geschlecht oder das Alter des Patienten, die Art, Dauer und Dominanz seiner Erkrankung sowie andere Faktoren wie die soziale Anerkennung oder die diametralen Persönlichkeitseigenschaften Introversion-Extroversion nach Hans Jürgen Eysenck [7]. Erstaunlicherweise korrelierten selbst die Suggestibilität, Hypnotisierbarkeit sowie die Absorptionsfähigkeit, d.h. die Fähigkeit, sich in die Gedanken anderer zu versetzen, nicht oder nur sehr geringfügig mit dem Ausmaß eines Placeboeffekts.

Korrelationen Dementgegen wurden signifikante Korrelationen zwischen der Ausprägung einer PlaceboHypalgesie und der Angst eines Patienten entdeckt. Je mehr Angst ein Patient z. B. vor Schmerzen hatte, desto ausgebildeter war auch die Placebo-Hypalgesie. Neben den Angstwerten sind auch andere patientenspezifische Faktoren relevant für die Reduktion der Schmerzwahrnehmung. So sind der Optimismus eines Patienten und dessen Motivation zur Therapie ebenso bedeutsam wie sein Glaube, die richtige Medizin zu erhalten. Als weitere Faktoren gelten die allgemeine Konditionierbarkeit des Patienten sowie seine Vorerfahrungen mit Pharmakotherapien. Und schließlich beeinflusst auch die Anziehungskraft des Therapeuten, d.h. seine Attraktivität, Kompetenz und seine Liebenswürdigkeit, einen Placeboeffekt.

Sichtweise des Patienten Howard Brody nannte die „Bedeutung, die der Patient der Krankheit beimisst“ als weiteren wichtigen Faktor, der drei generelle Unterfaktoren beinhaltet [8]:

1. verständliche und zufriedenstellende Erklärung der Erkrankung durch den Behandler

2. ausreichendes Mitgefühl und adäquate Versorgung

3. Glaube des Patienten an die Überwindung der Symptome oder zumindest deren Kontrolle

Entscheidend für die Wirkung dieser drei Unterfaktoren ist ausschließlich die Sichtweise des Patienten. Wenn seine Anschauungen positiv beeinflusst werden, können positive Heilungseffekte generiert werden.

\section{Generierung positiver Heilungseffekte}

Konditionierung Positive Heilungseffekte lassen sich aktiv fördern [9]. Dies gelingt mit einfachen Umgebungsvariablen wie etwa einer sauberen Arbeitskleidung und einem gepflegten Erscheinungsbild, einem ordentlichen Wartebereich, einem gutem System zur Terminvergabe, kurzen Wartezeiten sowie ausreichenden Parkmöglichkeiten. Diese zum Teil leicht realisierbaren Details tragen zu einem Placeboeffekt bei, weil sie ihre Wirkung u.a. über Konditionierungsprozesse entfalten. Wenn ein Patient zum wiederholten Male eine Praxis mit ansprechendem Ambiente und freundlichen Mitarbeitern besucht, wirkt dies wie ein konditionierter Stimulus.

\section{Strategien}

Noch wichtiger: Die bei der Anamnese gezeigte Warmherzigkeit und das demonstrative Mitgefühl des Therapeuten sind maßgeblich für die Zufriedenheit und Compliance des Patienten. Dies wiederum ist von entscheidender Bedeutung für positive Heilungseffekte.

Lege artis können vier Strategien genutzt werden, um Placeboeffekte zur Unterstützung der Therapie zu generieren. Geeignet sind kognitive, affektive, verhaltenstherapeutische und soziale Strategien. Zu jeder dieser Strategien lassen sich Unterpunkte differenzieren $[10,11]$.

Training Einige US-amerikanische und deutsche medizinische Fach- und Hochschulen bieten spezielle Trainingsprogramme an, um den Umgang mit diesen Strategien zu erlernen und spezifisch zu nutzen $[12,13]$.

\section{Kognitive Strategien}

Fragen Sie Ihren Patienten nach seinem Erklärungsbild für seine Krankheit und lassen Sie ihn wenn möglich ausreden. Über diesen Weg finden Sie schnell einen Zugang, um die drei von Brody postulierten Unterfaktoren - Erklärung der Erkrankung, Mitgefühl und Versorgung sowie Glaube an die Überwindung/Kontrolle der Symptome positiv zu beeinflussen.

Nur wenn ein Therapeut weiß, wie ein Patient seine Erkrankung deutet, kann er dessen Erklärung bzw. sein Krankheitsmodell modifizieren. Dadurch gelingt es, (1) das Verständnis des Patienten für seine Erkrankung zu verbessern, (2) ihm die Art und das Ausmaß der Versorgung mit Blick auf die Entstehung und den Verlauf seiner Krankheit verständlicher zu machen, (3) seine positiven Erwartungen hinsichtlich der Therapie zu verstärken und (4) seinen Glauben an die Überwindung oder Kontrolle der Symptome zu bekräftigen. 
Therapieerwartungen Zu den kognitiven Strategien gehören außerdem die Verhandlung und unter Umständen auch die Umwandlung von Prioritäten und Therapieerwartungen. Unrealistische Therapieerwartungen führen zwangsläufig zu Enttäuschung und Frustration. Demzufolge ist es elementar, die an die Behandlung geknüpften Erwartungen frühzeitig und explizit zu erfragen. Sind die Erwartungen des Patienten unrealistisch, erläutern Sie ihm hierfür die Gründe. Bilden Sie dazu den Patienten weiter, und legen Sie ihm seine Prognose dar. Nutzen Sie Erklärungsmodelle mit bildlichen Darstellungen, um ihm zu veranschaulichen, warum seine Erwartungen an die Therapie wenig realistisch sind.

Systematische Untersuchungen unter Praxisbedingungen ergaben eine erstaunlich geringe Übereinstimmung der Behandlungsziele und -erwartungen von Therapeuten und Patienten. Oftmals hegen Patienten versteckte Erwartungen, welche nicht aus ihrer vordergründigen Symptomatik heraus verstanden werden können. Die angeregte Einbindung eines Patienten in Therapieentscheidungen verbessert diese Situation deutlich. Folglich ist es zweckdienlich, einen Patienten bereits in der Anamnese nach seinen Erwartungen zu befragen und diese gegebenenfalls mit ihm zu verhandeln.

\section{„Treffen Sie Übereinkünfte zu Zielen, Methoden und Bedingungen der Therapie! “}

Der Konsens über gemeinsame Behandlungsziele führt zur Wahrnehmung höherer Kompetenz, er steigert die Verantwortlichkeit beider Seiten für den Therapieerfolg, und er schützt vor unrealistischen Wunschvorstellungen, deren Nichteintreten einen bereits errungenen Teilerfolg subjektiv negativ bewerten lässt.

Shared-Decision-Making Ein wesentliches Element der kognitiven Strategie ist das Offerieren von Entscheidungsmöglichkeiten. Mit Blick auf gemeinsame Therapieziele kann ein Patient in die Auswahl geeigneter Übungen miteinbezogen werden. Das Darlegen von Entscheidungsmöglichkeiten muss aber nicht zwingend Bestandteil jeder Behandlung sein. Es gibt sehr häufig Situationen, in denen die Therapie vom Patienten eine erhebliche Veränderung seiner Lebensgewohnheiten erfordert. Um dies zu ermöglichen, sollten unbedingt Alternativen für unterschiedliche Szenarien erörtert werden. Dadurch steigt die Akzeptanz des Patienten für die Therapiemaßnahme erheblich.

Edukation Zu den Bestandteilen der kognitiven Strategie gehört nicht zuletzt auch die Weiterbildung des Patienten. Es wurde wiederholt gezeigt, dass die Patientenedukation die Wirkung von Bewältigungsstrategien verbessert, die Therapiezufriedenheit steigert und die Compliance erhöht.

\section{Affektive Strategien}

Affektive Strategien sind auf den Abbau negativer Emotionen, besonders von Angst, Depression und Ärger, ausgerichtet. Der Umgang mit diesen Strategien kann erlernt bzw. deutlich verbessert werden [12-14].

Zu den affektiven Strategien zählen u. a. das Einfühlungsvermögen und der Zuspruch des Behandlers, das Vermitteln von Hoffnung, das Beruhigen sowie das Ermuntern des Patienten zur Darstellung seines emotionalen Zustandes und das Verstärken der Selbstversöhnung.

\section{„Ich verstehe gut, wie schwer es Ihnen fallen muss, mit Ihrer Krankheit zu leben."}

Das Einfühlungsvermögen ist eine potente therapeutische Intervention. Bereits mit einfachen Aussagen kann die Interaktion zwischen Patient und Therapeut verbessert werden, um schneller das Therapieziel zu erreichen.

\section{Verhaltenstherapeutische Strategien}

Verhaltenstherapeutische Strategien sollen fehladaptierte Verhaltensrituale und/oder Bewegungsstereotype dauerhaft ändern. Der Schwerpunkt liegt auf der Betonung der aktiven Rolle des Klienten. Die Erhöhung der Aktivität verbessert die Kontrollmöglichkeiten eines Patienten und stärkt seine Kontrollüberzeugungen. Er fühlt sich dann wieder oder besser in der Lage, seine Symptomatik kontrollieren zu können.

Die verhaltenstherapeutischen Strategien umfassen auch das Aufzeigen alternativer Bewältigungsstrategien sowie das aufmerksame Verfolgen und Loben des gewünschten Verhaltens.

\section{Soziale Strategien}

Soziale Strategien unterstützen die vorgenannten Strategien. Sie zielen auf die Einbeziehung der Familie des Patienten und seines sozialen Netzwerks ab. Durch familiäres Engagement kann die häusliche Aktivität gesteigert werden. Andererseits können evtl. vorhandene operante Mechanismen, welche Schmerzen und/oder Inaktivität fördern, aufgedeckt und abgebaut werden. Angenehme Aktivitäten mit Partnern oder Kindern können zur Genesung beitragen, indem sie das allgemeine Aktivitätsniveau und Bewegungsverhalten verbessern.

\section{Fazit}

Verschiedene therapeutische Strategien sind geeignet, um die Bedeutung einer Behandlungsmaßnahme zu unterstreichen und zu verstärken. Gemäß der Placebo-Definition des NIH ist die Bedeutung, welcher der Patient einer Behandlungsmaßnahme zuspricht, von erheblicher Relevanz für das Ausmaß zusätzlicher positiver Heilungseffekte. 
Mittlerweile liegen zahlreiche Studien vor, welche positive Heilungseffekte mit Blick auf die Wirkung verschiedener Einflussgrößen aufdecken. Sämtliche Faktoren wirken letztendlich dadurch, dass sie den Bedeutungsgehalt der Therapie verändern. Hierzu gehört die weiße Kleidung medizinischen Fachpersonals, die Bewegungsgeschwindigkeit eines Therapeuten (hektisch vs. ruhig), seine Aussprache, die Art der Untersuchung (therapeutisch vs. experimentell) sowie gegebenenfalls auch spezifische kulturelle Einflüsse [15-18].

„Bedeutungseffekte“ Infolge der Studienlage ist eine Diskussion entbrannt, ob man Placeboeffekte nicht besser als „Meaning Response“ bzw. „Bedeutungseffekte“ bezeichnen sollte. Wahrscheinlich wird sich diese Begrifflichkeit nicht durchsetzen - auch weil dadurch Konditionierungsprozesse nur schwer zu fassen sind. Alles in allem ist es zwingend notwendig und an der Zeit, Placeboeffekte breiter zu definieren als nur Effekte einer scheinbar wirkungslosen Medikation. Vielmehr kann man eine Therapie lege artis durch Placeboeffekte zum Wohle des Patienten bedeutsam unterstützen. Dieses hilfreiche Add-on ist keine eigenständige Therapieform, sondern entfaltet seine Wirkung im Schulterschluss mit spezifischen und fundierten Behandlungsmaßnahmen.

\section{Autorinnen/Autoren}

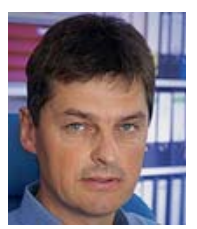

Thomas Weiß ist Facharzt für Physiologie (Neurophysiologie) und Professor am Lehrstuhl für klinische Psychologie der FriedrichSchiller-Universität Jena. Er betreibt intensive Grundlagenforschung zum Thema Schmerz, beispielsweise zur Bedeutung der Sprache für die Schmerzwahrnehmung.

Korrespondenzadresse

\author{
Thomas Weiß \\ Friedrich-Schiller-Universität Jena \\ Klinische Psychologie \\ Am Steiger 3 - Haus 1 \\ 07743 Jena \\ Deutschland \\ thomas.weiss@uni-jena.de
}

\section{Literatur}

[1] Stuhlreyer J, Klinger R. Klinische Aspekte von Placebo. Der Schmerzpatient 2020; 4: 172-178. doi:10.1055/a-12055303

[2] Luomajoki H, Morf R. Placeboeffekte in wissenschaftlichen Studien. Der Schmerzpatient 2020; 4: 166-170. doi:10.1055/ a-1205-5291

[3] Taxer B, Luomajoki H. Context matters: Placebos im Praxisalltag. Der Schmerzpatient 2020; 4: 184-190. doi:10.1055/ a-1205-5337
[4] Guess HA, Kleinman A, Kusek JW et al. The Science of the Placebo: Toward an Interdisciplinary Research Agenda. London: BMJ Books; 2002

[5] National Center for Complementary and Integrative Health. Placebo Effect. Im Internet: https://www.nccih.nih.gov/ health/placebo-effect; Stand: 20.07.2020

[6] Eippert F. Neurobiologische Grundlagen der Placebo-Analgesie. Der Schmerzpatient 2020; 4: 158-165. doi:10.1055/a1205-5280

[7] Weiss T. Multiple Hirnnetzwerke modulieren chronische Rückenschmerzen. Der Schmerzpatient 2020; 4: 153-156. doi:10.1055/a-1241-5713

[8] Brody $\mathrm{H}$. The doctor as therapeutic agent: a placebo effect research agenda. In: Harrington A, Hrsg. The Placebo Effect. Cambridge MA: Harvard University Press; 1999: 77-92

[9] Novack DA. Therapeutic aspects of the clinical encounter. Journal of General Internal Medicine 1987; 2: 346-355. doi:10.1007/BF02596174.

[10] Weiss T. Psychophysiologische Aspekte des Placeboeffekts bei Schmerz. Schlussfolgerungen für die Patient-TherapeutInteraktion. Zeitschrift für Neuropsychologie 2004; 15: 99110. doi:10.1024/1016-264X.15.2.99

[11] Weiss T. Plazebo-Effekte in der Physiotherapie. Physiopraxis 2009; 6: 3-13

[12] Novack DA, Suchman AL, Clark W et al. Calibrating the physician. Personal awareness and effective patient care. JAMA 1997; 278: 502-509. doi:10.1001/jama.278.6.502

[13] Dube CE, O'Donnell JF, Novack DA. Communication skills for preventive interventions. Academic Medicine 2000; 75: S4554. doi:10.1097/00001888-200007001-00007

[14] Novack DA, Epstein RM, Paulsen RH. Toward creating physican-healers: Fostering medical students' slef-awareness, personal growth and well-being. Academic Medicine 1999; 74: 516-520. doi:10.1097/00001888-199905000-00017

[15] Moerman DE. Explanatory mechanisms for placebo effects: cultural influences and the meaning response. In: Guess HA, Kleinman A, Kusek JW et al, Hrsg. The Science of Placebo Toward an interdisciplinary research agenda. London: BM] Books; 2002: 77-107

[16] Moerman DE. The meaning response: Thinking about placebos. Pain Pract 2006; 6: 233-236. doi:10.1111/j.15332500.2006.00091.x

[17] Moerman DE, Harrington A. Making space for the placebo effect in pain medicine. Sem Pain Med 2005; 3: 2-6. doi:10.1016/j.spmd.2005.02.008

[18] Moerman DE, Jonas WB. Deconstructing the placebo effect and finding the meaning response. Annals of Internal Medicine 2002; 136: 471-476. doi:10.7326/0003-4819-136-6200203190-00011

\section{Bibliografie}

Der Schmerzpatient 2020; 3: 179-182

DOI 10.1055/a-1205-5315

ISSN 2512-6210

(C) 2020. Thieme. All rights reserved.

Georg Thieme Verlag KG, Rüdigerstraße 14,

70469 Stuttgart, Germany 